\title{
Estudo dos componentes lipídicos das sementes de três espécies do gênero Cordia L. (Boraginaceae)
}

\author{
Arrebola, M.R.B.'; Peterlin, M.F.2; Bastos, D. H. M."; Rodrigues, R. F. de O..'; \\ Carvalho, P. de O..1* \\ ${ }^{1}$ Programa de Pós-Graduação em Ciências Farmacêuticas, Universidade São Francisco; ${ }^{2}$ Aluna de \\ Iniciação Científica PIBIC/CNPq da Universidade São Francisco, Bragança Paulista, SP; ${ }^{3}$ Departamento de \\ Nutrição, Faculdade de Saúde Pública, Universidade de São Paulo, São Paulo, SP.
}

Recebido para publicação em: 29/10/2003 Aceito para publicação em: 10/03/2004

RESUMO: Sementes de três espécies de Cordia L., da família Boraginaceae, coletadas em diferentes cidades do Estado de São Paulo foram analisadas quanto ao teor de lipídeos e composição de ácidos graxos, com ênfase na presença do ácido gama-linolênico, um ácido graxo de grande interesse terapêutico. Os lipídeos totais foram fracionados em classes (lipídeos neutros, fosfolipídeos e glicolipídeos) através de cromatografia em coluna aberta de gel de sílica e a composição de ácidos graxos foi determinada por cromatografia a gás. As concentrações de lipídeos totais apresentaram grande variação entre as amostras (25,84 a 62,81\%), sendo que a classe lipídica dominante foi de lipídeos neutros (média de $88,9 \%$ dos lipídeos totais). Os ácidos insaturados representaram $75 \%$ da composição total dos ácidos graxos, sendo o principal representante o ácido oléico. Verificou-se a presença do ácido gama-linolênico em todas as amostras analisadas, em concentrações que variaram de 0,63 a 2,54 \%, valores superiores àqueles relatados pela literatura para outras espécies do gênero Cordia $\mathrm{L}$.

Unitermos: Cordia; Boraginaceae; ácido gama-linolênico; óleo; sementes

\begin{abstract}
Lipids compounds study from seeds of three Cordia L. species (Boraginaceae). Seeds of three Cordia L. species (Boraginaceae), collected in different cities of the State of São Paulo were analysed in relation to the lipid content and fatty acid composition, with emphasis on the presence of gamma-linolenic acid, a fatty acid with therapeutical interest. The total lipids were separated into classes (neutral lipids, phospholipids and glucolipids) by open column chromatography with silica gel and the fatty acid composition was determined by gas chromatography. Although the levels of total lipids varied considerably between samples ( 25.84 to $62.81 \%$ ), the dominant class was that of neutral lipids ( $88.9 \%$ of the total lipids). The insaturated acids represented $75 \%$ of the total composition of fatty acids, with oleic acid being the main representative. The presence of gamma-linolenic acid was ascertained in all the samples analysed, in concentrations from 0.63 to $2.54 \%$, higher values than those related in literature for other Cordia.
\end{abstract}

Key words: Cordia; Boraginaceae; gamma-linolenic acid; seed oil 


\section{INTRODUÇÃO}

O gênero Cordia L. foi descrito por Linnaeus em 1753 (TARODA, 1984) e pertence a família Boraginaceae, a qual conta com cerca de 100 gêneros e 2.000 espécies difundidas nos trópicos, nas regiões temperadas e árticas (ANGELY, 1970; BARROSO, 1986; FICARRA et al., 1995). O gênero Cordia L. é representado por 26 espécies somente no Estado de São Paulo, e têm sido usado na medicina popular, principalmente no tratamento de úlceras gástricas, como antiinflamatórias e no tratamento de obesidade. Conhecida popularmente como "erva baleeira", Cordia curassavica (Jacq.) Roem. \& Schult. (=Cordia verbenaceae DC.), ocorre no Brasil, da região da Amazônia ao Rio Grande do Sul, preferencialmente de 500 a 1000 metros do litoral (ANGELY, 1970; SMITH, 1970). Costuma ser utilizada empiricamente no tratamento de tumores e úlceras, sendo ainda utilizadas como anti-reumática e hemostática (AKISUE et al., 1983). Cordia ecalyculata Vell. (=Cordia salicifolia Cham.), conhecida como porangaba e chá-de-bugre, tem sido empregada como diurética e no tratamento da obesidade (BARROSO et al., 2002). No Brasil, esta espécie vegetal ocorre desde Minas Gerais até o Rio Grande do Sul, sendo encontrada ainda em Brasília e no Acre. Outras espécies são ainda utilizadas como alimento, como é o caso de Cordia myxa L., incluída na seção Myxa (ANGELY, 1970). Apesar de não ser uma espécie nativa, existem relatos do uso de Cordia myxa L. na medicina popular, como laxante e como calmante da tosse em doenças pulmonares (SAITO; OLIVEIRA, 1984).

Estas plantas ainda são pouco estudadas do ponto de vista químico, apesar de muitas espécies apresentarem grande interesse como fonte de substâncias para a indústria farmacêutica ou de cosméticos. As principais substâncias encontradas nesta família são alcalóides, quinonas, naftoquinonas, saponinas, taninos, ácidos fenólicos, alantoínas, mucilagens, polissacarídeos, flavonóides, ciclitóis e ácidos graxos de interesse terapêutico e nutricional, como o ácido gamalinolênico (FELL; PECK, 1968; VELASCO; GOFFMAN, 1999; KUROYANAGI et al., 2001).

O ácido gama-linolênico, um ácido graxo triinsaturado da família ômega 6 (w6 ou n-6), tem recebido considerável atenção nos últimos anos devido aos efeitos benéficos relatados no tratamento e prevenção de doenças cardiovasculares (HORNYCH et al., 2002), diabetes (SURESH; DAS, 2003), eczema atópico (GOOL et al., 2003), alcoolismo (MEEHAN et al., 1995), síndrome pré-menstrual (GIRMAN et al., 2003), tratamento da hipertensão ( LOUIS et al., 1992; BELLENGERGERMAIN et al., 2002) e agente antitumoral (BAKSHI et al., 2003). Para este ácido também foi relatada ação hipocolesterolêmica, sendo capaz de diminuir os níveis de VLDL (lipoproteína de muito baixa densidade) e LDL (lipoproteína de baixa densidade) colesterol (FUKUSHIMA et al., 1996; LAIDLAW; HOLUB, 2003).

Preparações à base de óleo contendo ácido gama-linolênico obtido de fontes vegetais, como por exemplo, o óleo de prímula (Oenothera biennis L.), o óleo das sementes de borragem (Borago officinalis L.) e o óleo das sementes de groselha-negra (Ribes nigrum L.), são comercializadas em vários países, inclusive no Brasil, com os nomes de Prímuris ${ }^{\circledR}$ (Herbarium), Gamaline- $\mathrm{V}^{\circledR}$ (Herbarium), Primolan ${ }^{\circledR}$ (Bionatus), Borag ${ }^{\circledR}$ (Ativus), Livten ${ }^{\circledR}$ (Ativus), entre outros. Várias espécies de plantas das famílias Boraginaceae, Aceraceae e Ranunculaceae são conhecidas fontes de ácido gama-linolênico (WOLF et al., 1983).

A falta de informações disponíveis sobre as substâncias químicas, em especial os ácidos graxos encontrados nas espécies de Cordia, aliada à crescente oferta no comércio brasileiro de cápsulas gelatinosas contendo o ácido gama-linolênico, despertou o interesse em analisar a composição lipídica das sementes de espécies de Cordia encontradas no Brasil. Portanto, o objetivo deste trabalho é determinar o teor de lipídeos totais, as principais classes de lipídeos presentes no óleo das sementes, assim como a porcentagem do ácido gama-linolênico em relação ao teor total de ácidos graxos do óleo de quatro amostras de sementes de plantas maduras de Cordia, obtidas de diferentes regiões do Estado de São Paulo.

Rev. Bras. Farmacogn., V. 14, n. 1, jan.-jun. 2004. 


\section{MATERIAL E MÉTODOS}

\section{Meterial vegetal}

A identificação botânica das espécies analisadas foi realizada com a colaboração do Prof. Fernando de Oliveira (Universidade São Francisco). Excicatas representativas do material coletado acham-se depositadas no Herbário HRCB em Rio Claro e no Instituto Butantã - Horto Oswaldo Cruz em São Paulo.

\section{Preparo das amostras}

Foram analisadas amostras de sementes de plantas maduras: Cordia ecalyculata (duas amostras de regiões diferentes do Estado de São Paulo), Cordia myxa e Cordia sellowiana, coletadas de julho a setembro em diferentes regiões do Estado de São Paulo, conforme especificações apresentadas na Tabela 1. As sementes foram trituradas e homogeneizadas com auxílio de um multiprocessador e o pó obtido foi usado para as análises posteriores. As análises foram realizadas em triplicata e o resultado expresso pela média e desvio padrão das determinações.

Tabela 1. Dados sobre as amostras analisadas das sementes de algumas espécies de Cordia L., coletadas em diferentes cidades do Estado de São Paulo.

\begin{tabular}{l||c|c|}
\hline \multicolumn{1}{|c|}{ Amostras } & Local da coleta & Data da coleta \\
\hline Cordia ecalyculata & $\begin{array}{c}\text { Rio Claro } \\
\text { (Horto da Unesp) }\end{array}$ & setembro 2001 \\
\hline Cordia ecalyculata & $\begin{array}{c}\text { Guarulhos } \\
\text { (Faculdade de Guarulhos) }\end{array}$ & julho/agosto 2001 \\
\hline Cordia myxa & $\begin{array}{c}\text { Rio Claro } \\
\text { (Horto da Unesp) }\end{array}$ & agosto/setembro 2001 \\
\hline Cordia sellowiana & $\begin{array}{c}\text { Bragança Paulista } \\
\text { (Sítio Kadu - margem da represa) }\end{array}$ & setembro 2001 \\
\hline
\end{tabular}

\section{Extração e determinação do teor de lipídeos totais}

Foram utilizados $20 \mathrm{~g}$ do pó obtido para a extração a frio dos lipídeos totais, de acordo com o método de Bligh e Dyer (1959), levando-se em conta as proporções recomendadas entre os solventes clorofórmio, metanol e água destilada, 1:2:0,8 para a primeira diluição e 2:2:1,8 para a segunda diluição. O extrato clorofórmico obtido foi filtrado em papel de filtro, utilizando sulfato de sódio anidro e, em seguida, $10 \mathrm{~mL}$ do extrato foram transferidos para um béquer pré-tarado e colocado em estufa a $105^{\circ} \mathrm{C}$ até peso constante. Após resfriamento em dessecador, o béquer foi pesado em balança analítica obtendo-se o conteúdo de lipídeos totais expresso em g/Kg de amostra úmida. O restante do extrato clorofórmico foi concentrado em evaporador rotatório a $40{ }^{\circ} \mathrm{C}$, transferido para frasco âmbar e estocado $\mathrm{a}-30^{\circ} \mathrm{C}$ para análise posterior.

\section{Separação das classes de lipídeos por cromatografia em coluna}

Foi adotado o procedimento descrito por Christie (1982) para separação dos lipídeos totais (1,57 a 1,79 g) em classes de lipídeos, utilizando uma coluna de vidro (20 cm de comprimento e 
$1,25 \mathrm{~cm}$ de diâmetro interno) contendo $20 \mathrm{~g}$ de gel de sílica 60 (70-230 mesh, Merck $\left.{ }^{\circledR}\right)$ como $^{2}$ adsorvente e $200 \mathrm{~mL}$ de clorofórmio, acetona e metanol para eluição das três frações, lipídeos neutros ( $L N)$, glicolipídeos $(G L)$ e fosfolipídeos (FL), respectivamente. Após evaporação dos solventes em evaporador rotatório a $40^{\circ} \mathrm{C}$, as frações foram transferidas para frascos pré-tarados e o volume residual evaporado em atmosfera de $\mathrm{N}_{2}$, sendo então colocados em dessecador até peso constante. As porcentagens das classes de lipídeos foram calculadas em relação ao peso total dos lipídeos neutros e também expressos em relação ao peso da amostra úmida $(\mathrm{g} / \mathrm{Kg})$.

\section{Preparo de ésteres metílicos de ácidos graxos por derivatização}

Os lipídeos totais foram convertidos em ésteres metílicos de ácidos graxos com o reagente esterificante $\mathrm{BF}_{3} /$ metanol, de acordo com o método preconizado pela American Oil Chemist's Society (AOCS, 1993). Os ésteres metílicos diluídos em hexano foram analisados por cromatografia gasosa.

\section{Determinação da composição de ácidos graxos por cromatografia gasosa}

Foi utilizado o cromatógrafo $\mathrm{CHROMPACK}^{\circledR}$ modelo CP 9001 com detector de ionização de chama e coluna capilar CP-Sil 88 (Chrompack, WCOT Fused Silica $50 \mathrm{~m} \times 0,25 \mathrm{~mm}$ ). A temperatura do detector foi $270^{\circ} \mathrm{C}$, a do injetor $250^{\circ} \mathrm{C}$, e a temperatura inicial da coluna de $80^{\circ} \mathrm{C}, 7$ minutos, com programação de $10^{\circ} \mathrm{C} / \mathrm{min}$ até a temperatura de $180^{\circ} \mathrm{C}$ por 3 minutos, com propagação de $3^{\circ} \mathrm{C} / \mathrm{min}$ até a temperatura máxima de $210^{\circ} \mathrm{C}$. O gás de arraste foi o hidrogênio com fluxo de $2,0 \mathrm{~mL} / \mathrm{min}$. A identificação dos ácidos graxos foi realizada por comparação do tempo de retenção dos componentes da amostra com os de padrões autênticos de ésteres de ácidos graxos injetados nas mesmas condições. Foi utilizada também a técnica de coeluição ("spiking") de padrões junto com a amostra. A área dos picos foi calculada por meio de integrador (Chromato-Integrator) e expressa em porcentagem relativa de cada ácido graxo em relação aos ácidos graxos totais.

\section{RESULTADOS E DISCUSSÃO}

As porcentagens de lipídeos totais, determinadas pelo método gravimétrico, estão apresentadas na Tabela 2, e variaram de 25,84 $\pm 0,62 \mathrm{~g} / \mathrm{Kg}$ (Cordia ecalyculata coletada em Guarulhos) a 62,81 \pm $0,84 \mathrm{~g} / \mathrm{Kg}$ (Cordia myxa). Os valores de lipídeos totais obtidos para as duas amostras de Cordia ecalyculata foram bem diferentes, sendo que a amostra coletada em Rio Claro apresentou quase o dobro de lipídeos $(42,82 \pm 0,91 \mathrm{~g} / \mathrm{Kg})$ da coletada em Guarulhos $(25,84 \pm 0,62 \mathrm{~g} / \mathrm{Kg})$.

Tabela 2. Conteúdos de lipídeos totais das amostras analisadas das sementes de algumas espécies de Cordia L., obtidos por gravimetria.

\begin{tabular}{l|c}
\multicolumn{1}{c|}{ Amostras } & $\mathbf{g / K g}$ de amostra úmida ${ }^{(\mathbf{a})}$ \\
\hline Cordia ecalyculata $^{(\mathrm{b})}$ & $42,82 \pm 0,91$ \\
\hline Cordia ecalyculata $^{(\mathrm{c})}$ & $25,84 \pm 0,62$ \\
\hline Cordia myxa & $62,81 \pm 0,84$ \\
\hline Cordia sellowiana & $58,44 \pm 0,62$ \\
\hline
\end{tabular}

(a) Resultados expressos como média e desvio padrão das medidas realizadas em triplicata; (b) Amostra de Rio Claro; ${ }^{(c)}$ Amostra de Guarulhos

Rev. Bras. Farmacogn., V. 14, n. 1, jan.-jun. 2004. 
Os valores acham-se próximos daqueles relatados por Kleiman et al. (1964) para as sementes de Cordia obliqua Wild. (40 g/Kg) e por Miller et al. (1967) para as sementes de Cordia verbenacea DC. (=Cordia curassavica (Jacq.) Roem. \& Schult.) $(24,4 \mathrm{~g} / \mathrm{Kg}$ ). As sementes da espécie Cordia ecalyculata (=Cordia salicifolia Cham.), analisadas por Miller et al. (1967) apresentaram o teor médio de lipídeos totais de 67,0 g/Kg de amostra. Em contrapartida, valores superiores de lipídeos totais foram relatados por outros autores para outras espécies de Cordia L. Daulatabad et al. (1992) relataram $180 \mathrm{~g} / \mathrm{Kg}$ para a espécie Cordia rothii Roem.\& Schult., enquanto a média de lipídeos relatada por Mayworm et al. (1998) para as sementes de Cordia glabrata A. DC., da região semi-árida do nordeste brasileiro, foi de $196 \mathrm{~g} / \mathrm{Kg}$.

Algumas plantas da família Boraginaceae são conhecidas pelos altos teores de lipídeos. Merecem destaque os valores relatados para as espécies Myosotis discolor Pers. (34,1\%) e Myosotis sylvatico Hoffm. (31,8 \%) (VELASCO; GOFFMAN, 1999). Guil-Guerrero et al. (2001), analisaram 20 espécies da família Boraginaceae coletadas na Espanha, e relataram teores de lipídeos de 6,63\% (Echium humile ) a 30,97\% (Borago officinalis). Os autores citados acima não analisaram nenhuma espécie do gênero Cordia L.

Na Tabela 3 estão apresentados os conteúdos das frações de lipídeos neutros (LN), glicolipídeos (GL) e fosfolipídeos (FL) das espécies de sementes de Cordia L. Os LN são a classe majoritária, contribuindo com 86,41 a 92,91 \% dos lipídeos totais, seguida dos FL com 6,05 a 8,87 $\%$ e dos GL com 1,04 a 4,72\%. Não existem dados disponíveis na literatura, até a presente data, relativos às frações de lipídeos de Cordia L. Senanayake e Shahidi (2000) encontraram teores médios de $84,1 \%$ de $L N, 8,6 \%$ de $F L$ e $7,3 \%$ de GL, após 10 dias da germinação das sementes de borragem (Borago officinalis L.), também pertencente a família Boraginaceae. A ocorrência de glicolipídeos não foi registrada por Hamrouni et al. (2002) para esta mesma espécie.

Tabela 3. Conteúdo das frações de lipídeos das amostras analisadas das sementes de algumas espécies de Cordia L.

\begin{tabular}{|c|c|c|c|c|c|c|}
\hline \multirow{3}{*}{ Amostras } & \multicolumn{6}{|c|}{ Conteúdo das frações de lipídeos } \\
\hline & \multicolumn{3}{|c|}{ (\%) g/100 g lipídeos totais ${ }^{(a)}$} & \multicolumn{3}{|c|}{$\mathrm{g} / \mathrm{kg}$ amostra úmida } \\
\hline & LN & GL & PL & LN & GL & PF \\
\hline Cordia ecalyculata $^{(\mathrm{b})}$ & $88,64 \pm 0,33$ & $3,23 \pm 0,21$ & $8,13 \pm 0,24$ & 37,95 & 1,38 & 3,48 \\
\hline Cordia ecalyculata $^{(\mathrm{c})}$ & $92,91 \pm 0,42$ & $1,04 \pm 0,12$ & $6,05 \pm 0,22$ & 24,02 & 0,26 & 1,56 \\
\hline Cordia myxa & $87,79 \pm 0,28$ & $4,24 \pm 0,18$ & $7,97 \pm 0,26$ & 55,14 & 2,66 & 5,00 \\
\hline Cordia sellowiana & $86,41 \pm 0,26$ & $4,72 \pm 0,14$ & $8,87 \pm 0,24$ & 50,49 & 2,75 & 5,18 \\
\hline
\end{tabular}

(a) Resultados expressos como média e desvio padrão das medidas realizadas em triplicata; (b) Amostra de Rio Claro; ${ }^{\left({ }^{c}\right)}$ Amostra de Guarulhos; LN = lipídeos neutros; GL = glicolipídeos; FL = fosfolipídeos.

A Tabela 4 apresenta a composição relativa de ácidos graxos das espécies de sementes analisadas. Os resultados demonstraram que em todas as espécies analisadas predomina 0 ácido oléico (18:1 n-9), com aproximadamente $50 \%$ da composição total do óleo, seguido pelos ácidos linoléico (18:2 n-6) e palmítico (16:0); a menor porcentagem refere-se ao ácido palmitoléico (16:1 n-7). Aproximadamente $75 \%$ da composição dos ácidos graxos do óleo destas sementes é proveniente dos ácidos insaturados (mono e poliinsaturados). 
Tabela 4. Composição relativa dos principais ácidos graxos (\%) das amostras analisadas das sementes de algumas espécies de Cordia L. (a)

\begin{tabular}{|l|l|c|c|c|c|}
\hline \multicolumn{2}{|c|}{ Ácidos graxos } & $\begin{array}{c}\text { Cordia } \\
\text { ecalyculata }\end{array}$ & $\begin{array}{c}\text { Cordia } \\
\text { ecalyculata }\end{array}$ & $\begin{array}{c}\text { Cordia } \\
\text { myxa }\end{array}$ & $\begin{array}{c}\text { Cordia } \\
\text { sellowiana }\end{array}$ \\
\hline $10: 00: 00$ & Cáprico & - & $1,16 \pm 0,22$ & - & - \\
\hline $12: 0$ & Láurico & - & $2,57 \pm 0,18$ & - & - \\
\hline $14: 0$ & Mirístico & $1,36 \pm 0,12$ & $2,76 \pm 0,12$ & $0,45 \pm 0,13$ & $0,76 \pm 0,18$ \\
\hline $16: 0$ & Palmítico & $14,84 \pm 0,27$ & $16,48 \pm 0,21$ & $17,58 \pm 0,20$ & $10,95 \pm 0,24$ \\
\hline $16: 1$ n-7 & Palmitoléico & $0,76 \pm 0,12$ & $1,03 \pm 0,08$ & $0,35 \pm 0,05$ & $0,56 \pm 0,07$ \\
\hline $18: 0$ & Esteárico & $5,65 \pm 0,21$ & $5,73 \pm 0,14$ & $6,71 \pm 0,18$ & $6,08 \pm 0,30$ \\
\hline $18: 1$ n-9 & Oléico & $45,80 \pm 0,15$ & $47,08 \pm 0,18$ & $41,43 \pm 0,22$ & $54,70 \pm 0,23$ \\
\hline $18: 2$ n-6 & Linoléico & $27,66 \pm 0,31$ & $17,02 \pm 0,28$ & $29,98 \pm 0,25$ & $24,05 \pm 0,24$ \\
\hline $20: 0$ & Araquídico & $1,39 \pm 0,13$ & $1,68 \pm 0,14$ & $1,25 \pm 0,18$ & $1,09 \pm 0,23$ \\
\hline $18: 3 \gamma$ n-6 & Gama-linolênico & $1,10 \pm 0,12$ & $2,54 \pm 0,15$ & $0,63 \pm 0,12$ & $1,13 \pm 0,15$ \\
\hline $22: 0$ & Behênico & $1,42 \pm 0,18$ & $1,95 \pm 0,20$ & $1,13 \pm 0,12$ & $0,66 \pm 0,20$ \\
\hline $22: 1$ n-9 & Erúcico & - & - & $0,49 \pm 0,13$ & - \\
\hline
\end{tabular}

(a) Resultados expressos como média e desvio padrão das medidas realizadas em triplicata; ${ }^{(b)}$ Amostra de Rio Claro; (c) Amostra de Guarulhos.

Embora a predominância do ácido oléico também tenha sido relatada por Mayworm et al. (1998) para as sementes de Cordia glabrata A. DC. (40,8 \% de ácido oléico em relação aos ácidos graxos totais), oriundas do nordeste brasileiro, outras espécies de Cordia têm mostrado composições diferenciadas. Por exemplo, no óleo extraído de sementes de Cordia rothii Roem. \& Schult., o ácido predominante é o linoléico (40\%), seguido dos ácidos palmítico $(32,8 \%)$, ricinoléico $(10,8 \%)$ e oléico $(7,8 \%)$. O ácido alfa-linolênico $(24,1 \%)$ é predominante no óleo das sementes de Cordia myxa, no qual não foi identificado o seu isômero, o ácido gama-linolênico, conforme relatado por Mukarram et al. (1986).

Pelos dados da literatura pode-se perceber que as espécies pertencentes à família Boraginaceae mostram uma composição de ácidos graxos bem diversificada (VELASCO; GOFFMAN, 1999; GUIL-GUERRERO et al., 2001). Sabe-se que, diferentes condições, tais como qualidade do solo, estágio de germinação das sementes, chuva, temperatura e outros fatores ambientais, podem causar variações significativas, tanto no conteúdo de lipídeos, quanto na composição de ácidos graxos dos óleos obtidos de fontes vegetais (SENANAYAKE; SHAHIDI, 2000; RAHAMATALLA et al., 2001).

Nas quatro amostras analisadas de Cordia L. foi constatada a presença do ácido gamalinolênico, verificando-se uma relação inversa entre as concentrações deste ácido e de lipídeos totais entre as amostras analisadas. Por exemplo, nas amostras de Cordia ecalyculata, coletadas em Guarulhos e Rio Claro, as concentrações de lipídeos totais foram de 25,84 \pm 0,62 e 42,82 \pm $0,91 \mathrm{~g} / \mathrm{Kg}$, enquanto as concentrações de ácido gama-linolênico foram de 1,10 $\pm 0,12$ e 2,54 \pm $0,15 \%$, respectivamente, sugerindo que diferentes locais, condições de crescimento e época de coleta interferem significativamente na composição lipídica destas sementes.

Rev. Bras. Farmacogn., V. 14, n. 1, jan.-jun. 2004. 
As concentrações do ácido gama-linolênico variaram de 0,63 $\pm 0,12 \%$ (Cordia myxa) a $2,54 \pm 0,15 \%$ (Cordia ecalyculata coletada em Guarulhos), valores relativamente superiores aos relatados para diferentes espécies de Cordia L. A presença do ácido gama-linolênico não foi observada para as espécies de Cordia salicifolia (Cordia ecalyculata) e Cordia verbenacea (=Cordia curassavica) analisadas por Miller et al. (1967), e também para a Cordia rothii (DAULATABAD et al., 1992) e Cordia glabrataA. DC. (MAYWORM et al., 1998). Quantidades muito pequenas $(<0,1$ \%) foram relatadas para Cordia obliqua Willd. (KLEIMAN et al., 1964). Em contrapartida, o ácido gama-linolênico está presente em outras espécies da família Boraginaceae, em concentrações que variam de 0,7\% para Cerinthe major L. a 24,4\% para sementes de Borago officinalis L. (VELASCO; GOFFMAN, 1999).

Um fator de grande importância é a correlação entre a porcentagem de ácido gama-linolênico presente no óleo e a quantidade de óleo possível de ser obtida da fonte. Por exemplo, as espécies de sementes de Boraginaceae, analisadas por Velasco e Goffman (1999), das quais mereceu destaque o genêro Myosotis pelo alto conteúdo de óleo, contêm quantidades relativamente baixas de ácido gama-linolênico (média de $4 \%$ ). Em contrapartida, as sementes de Borago officinalis L. (borragem) apresentaram alto conteúdo de lipídeos (25,1\%) e também de ácido gama-linolênico $(24,4 \%)$. Por este motivo, o óleo extraído das sementes desta espécie tem sido usado para a fabricação de cápsulas contendo ácido gama-linolênico. O óleo de semente de prímula (Oenothera biennis L.), contendo 7 a $14 \%$ de ácido gama-linolênico (HUDSON, 1984) e o óleo das sementes de groselha-negra (Ribes nigrum L.), contendo 15 a $20 \%$ deste ácido graxo (TRAITLER et al.,1984; GOFFMAN; GALETTI, 2001) são outras fontes usadas comercialmente.

\section{CONCLUSÕES}

As espécies analisadas apresentaram concentrações relativamente baixas de lipídeos totais, quando comparadas com outras espécies de Boraginaceae, utilizadas industrialmente para obtenção de óleo contendo ácido gama-linolênico. Constatou-se também a presença do ácido gama-linolênico, um ácido graxo essencial da série ômega 6 (n-6), nas sementes das espécies Cordia ecalyculata, C. myxa e C. sellowiana, coletadas no Estado de São Paulo, em concentrações superiores àquelas relatadas na literatura para outras espécies do gênero Cordia L. Os dados relatados podem trazer subsídios aos consumidores brasileiros e também às indústrias farmacêuticas e alimentícias, que interessam-se pelos benefícios do ácido gama-linolênico, e comercializam alguns tipos de suplementos alimentares e alimentos enriquecidos com fontes deste ácido, respectivamente.

\section{AGRADECIMENTOS}

Os autores agradecem ao Prof. Dr. Fernando de Oliveira, do Laboratório de Pesquisa de Produtos Naturais da Universidade São Francisco de Bragança Paulista, pela identificação e fornecimento das sementes de Cordia utilizadas para a execução deste trabalho.

\section{REFERÊNCIAS}

ANGELY, J. Flora analítica e fitogeográfica do Estado de São Paulo. Phyton: International Journal of Experimental Botany, p.819-825, 1970.

AKISUE, M.K.; OLIVEIRA, F.; MORAES, M.S.; AKISUE, G.; MANCINI, B. Caracterização farmacognóstica da droga e da tintura de Cordia verbenaceaAl. DC. - Boraginaceae. Revista de Ciências Farmacêuticas, v.5, p.69-82, 1983. 
AMERICAN OIL CHEMIST'S SOCIETY (AOCS) Official methods and recommended practices of the American Oil Chemist's Society. 4.ed., Champaign, v.2, Ce 2-66, 1993.

BAKSHI, A.; MUKHERJEE, D.; BAKSHI, A.; BANERJI, A.K. DAS, U.N. $\gamma$-linolenic acid therapy of human gliomas. Nutrition, v.19, n.4, p.305-309, 2003.

BARROSO, G.M. Sistemática das angiospermas do Brasil. Viçosa: UFV, p. 88-94, 1986.

BARROSO, I.C.E.; OLIVEIRA, F. de; BRANCO, L.H.Z.; KATO, E.T.M.; DIAS, T.G. O gênero Cordia L.: botânica, química e farmacologia. Revista Lecta, v.20, n.1, p.15-34, 2002.

BELLENGER-GERMAIN, S.B.; POISSON, J.P.; NARCE, M. Antihypertensive effects of a dietary unsaturated FA mixture in spontaneously hypertensive rats. Lipids, v.37, n.6, p.561-567, 2002.

BLIGH, E.G.; DYER, J.W. A rapid method of total lipid extraction and purification. Canadian Journal of Biochemistry and Physiology, v.37, p. 911-917, 1959.

CHRISTIE, W.W. Analysis of complex lipid. In: Lipid Analysis, 2 ed., New York: Pergamon, p.107-134, 1982.

DAULATABAD, C.M.J.D; DESAI, V.A.; HOSAMANI, K.M. Unusual Fatty Acids of Cordia rothii seed oil. Journal of Science Food Agriculture, v.58, p.285-286, 1992.

FELL, K.R.; PECK, M.J. Phytochemical investigation of some species of the Boraginaceae. Planta Medica, v.16, n.4, p.411-420, 1968.

FICARRA, R.; FICARRA, P.; TOMMASINI, S. Leaf extracts of some Cordia species analgesic and antiinflammatory activities as well as their chromatographic analysis. Fármaco, v.50, p.245-256, 1995.

FUKUSHIMA, M.; AKIBA, S.; NAKANO, M. Comparative hypocholesterolemic effects of six vegetables oils in cholesterol-fed rat. Lipids, v.31, n.4, p.415-419, 1996.

GIRMAN, A.; LEE, R.; KLIGLER, B. An integrative medicine approach to premenstrual syndrome. American Journal Obstetrics and Gynecology, v.188, v.5 Suppl, p.S56-65, 2003.

GOFFMAN, F.D; GALLETTI, S. Gamma-linolenic acid and tocopherol contents in the seed oil of 47 accessions from several Ribes species. Journal of Agricultural and Food Chemistry, v.49, n.1, p.349-354, 2001.

GOOL, C.J.A.W.; THIJS, C.; HENQUET, C.J.M.; HOUWELINGEN, A.C.; DAGNELIE, P.C.; SCHRANDER, J.; MENHEERE, P.P.C.A.; BRANDT, P.A. $\gamma$-Linolenic acid supplementation for prophylaxis of atopic dermatitisa randomized controlled trial in infants at high familial risk. American Journal of Clinical Nutrition, v.77, n.4, p.943-951, 2003.

GUIL-GUERRERO, J.L.; MAROTO, F.F.G.; GIMÉNEZ,A.G. Fatty acids profiles from forty-nine plant species that are potential new sources of gamma-linolenic acid. The Journal of the American Oil Chemists' Society, v.78, n.7, p.677-684, 2001.

HAMROUNI, I.; TOUATI, S.; MARZOUK, B. Evolution des lipides au cours de la formation et de la maturation de la graine de bourrache (Borago officinalis). La Rivista Italiana delle Sostanze Grasse, v.79, p.113-118, 2002.

HORNYCH, A.; ORAVEC, S.; GIRAULT, F.; FORETTE, B.; HORROBIN, D.F. The effect of gamma-linolenic acid on plasma and membrane lipids and renal prostaglandin synthesis in older subjects. Bratislavske Lekarske Listy, v.103, n.3, p.101-7, 2002.

HUDSON, B.J.F. Evening Primose (Oenothera spp.) Oil and Seed. The Journal of the American Oil Chemists' Society, v.61, n.3, p.540-543, 1984.

KLEIMAN, R.; EARLE, F.R.; WOLFF, I.A. Search for New Industrial Oils. XI. Oils of Boraginaceae. The Journal of the American Oil Chemists' Society, v.41, p. 459-460, 1964.

KUROYANAGI, M.; SEKI, T.; HAYASHI, T.; NAGASHIMA, Y.; KAWAHARA, N.; SEKITA, S.; SATAKE, M. Anti-androgenic triterpenoids from Brazilian medicinal plants. Chemical and Pharmaceutical Bulletin, v.49, n.8, p.954-957, 2001.

LAIDLAW, M.; HOLUB, B.J. Effects of supplementation with fish oil-derived $n-3$ fatty acids and $\gamma$-linolenic acid on circulating plasma lipids and fatty acid profiles in women. American Journal of Clinical Nutrition, v.77, n.1, p.37-42, 2003.

Rev. Bras. Farmacogn., V. 14, n. 1, jan.-jun. 2004. 
LOUIS, C.S.; LEE, R.M.K.W; ROSENFELD, J.; BABJAK, A.F. Antihypertensive effect of $\gamma$-linolenic acid in spontaneously hypertensive rats. Hypertension, v.19, n.2, Supplement II, p.111-115, 1992.

MAYWORM, M.A.S.; NASCIMENTO, A.S.; SALATINO, A. Seeds of species from the "caatinga": proteins, oils and fatty acid contents. Revista Brasileira de Botânica, v. 21, n.3, p.299-303, 1998.

MEEHAN, E.; BEAUGÉ, F.; CHOQUART, D.; LEONARD, B.E. Influence of an n-6 polyunsaturated fatty acidenriched diet on the development of tolerance during chronic ethanol administration in rats. Alcoholism Clinical and Experimental Research, v.19, n.6, p.1441-6, 1995.

MILLER, R.W.; EARLE, F.R.; WOLFF, I.A. Search for New Industrial Oils. XV. Oils of Boraginaceae. Lipids, v.3, n.1, p.43 - 45, 1967.

MUKARRAM, M.; AHMAD, I.; FAROOQI, A. Studies on minor seed oils. VI. Fette Seifen Anstrichmittel, v.88, n.5, p.182-183, 1986.

RAHAMATALLA, A.B.; BABIKER, E.E.; KRISHNA, A.G.; TINAY, A.H. Changes in fatty acids composition during seed growth and physicochemical characteristics of oil extracted from four safflower cultivars. Plants Food for Human Nutrition, v.56, n.4, p.385-395, 2001.

SAITO, M.L.; OLIVEIRA, F. Morfodiagnose e identificação cromatográfica em camada delgada de chá de bugre - Cordia ecalyculata Vell. Revista Brasileira de Farmácia, v.67, p.1-16, 1986.

SENANAYAKE, N.S.P.J.; SHAHIDI, F. Lipid components of borage (Borago officinalis L.) seeds and their changes during germination. The Journal of the American Oil Chemists' Society, v.77, n.1, p.55-61, 2000.

SMITH, L.B. Boragináceas. In: REITZ, P.R. (ed.). Flora llustrada Catarinense. Itajaí: Herbário Barbosa Rodrigues, 1970, p.85.

SURESH, Y.; DAS, U.N. Long-chain polyunsaturated fatty acids and chemically induced diabetes mellitus Effect of $\omega-6$ fatty acids. Nutrition, v.19, n.2, p.93-114, 2003.

TARODA, N. Taxonomic studies on Brazilian species of Cordia L. (Boraginaceae) Ph. D. Thesis. University of St. Andrews, Scotland, 1984.

TRAITLER, H.; WINTER, H.; RICHLI, U.; INGENBLEEK, Y. Characterization of $\gamma$-Linolenic Acid in Ribes Seed. Lipids, v.19, n.12, p.923-928, 1984.

VELASCO, L.; GOFFMAN, F.D. Chemotaxonomic significance of fatty acids and tocopherols in Boraginaceae. Phytochemistry, v.52, p.423-426, 1999.

WOLF, R.B.; KLEIMAN, R.; ENGLAND, R.E. New Sources of $\gamma$-Linolenic Acid. The Journal of the American Oil Chemists'Society, v.60, n.11, p.1858-1860, 1983.

\section{Autor para correspondência:}

Profa. Dra. Patrícia de Oliveira Carvalho

Programa de Pós-Graduação em Ciências Farmacêuticas

Universidade São Francisco

Av. São Francisco de Assis, 218

CEP 12916900, Bragança Paulista, SP.

E-mail: patcarvalho@saofrancisco.edu.br 